\title{
Mechanical Researches on Young's Modulus of SCS Nanostructures
}

\author{
Qinhua Jin, Tie Li, Ping Zhou, and Yuelin Wang \\ State Key Laboratories of Transducer Technology, National Key Laboratory of Microsystem Technology, \\ Shanghai Institute of Microsystem and Information Technology, Shanghai 200050, China \\ Correspondence should be addressed to Yuelin Wang, ylwang@mail.sim.ac.cn
}

Received 4 December 2008; Accepted 13 February 2009

Recommended by Bohua Sun

\begin{abstract}
Nanostructures of SingleCrystalSilicon (SCS) with superior electrical, mechanical, thermal, and optical properties are emerging in the development of novel nanodevices. Mechanical properties especially Young's modulus are essential in developing and utilizing such nanodevices. In this paper, experimental researches including bending tests, resonance tests, and tensile tests on Young' $\mathrm{s}$ modulus of nanoscaled SCS are reviewed, and their results are compared. It was found that the values of $E$ measured by different testing methods cannot match to each other. As the differences cannot be explained as experimental errors, it should be understood by taking surface effect into account. With a simplified model, we qualitatively explained the difference in $E$ value measured by tensile test and by resonance test for Si nanobeams.
\end{abstract}

Copyright ( 2009 Qinhua Jin et al. This is an open access article distributed under the Creative Commons Attribution License, which permits unrestricted use, distribution, and reproduction in any medium, provided the original work is properly cited.

\section{Introduction}

Being the most important semiconductor used in microelectronics and microelectromechanical systems, Single Crystalline Silicon (SCS) is still a research hot spot in nanoscience and nanotechnology, as current trend pushes sciences and technologies to nanometer scale. Researchers have found that Si structures would exhibit unique electrical [1], mechanical [2], thermal [3], and optical [4] properties at nanoscale. This makes Si nanostructures (nanobeams and nanowires) attractive in developing Field-effect transistors (FETs), fieldemission devices, chemical sensors, nanoresonators, and photonics, and so forth. However, in order to make these devices reliable, mechanical properties, especially Young's modulus $(E)$ of Si nanostructures are essential and critical to study.

Being defined as the ratio of tensile stress to tensile strain, $E$ is always considered as an intrinsic material property and fundamentally related to internal atomic bonding. The stronger the atomic bonding is, the larger the Young's modulus is. Besides the direct testing method, tensile test, there are many other techniques for obtaining $E$, for example, bending/curvature, resonance, indentation, and so forth. But in nanoscale, it is in question that whether these testing methods are still effective, and where surface effect cannot be ignored.

In this review, we present the recent experimental researches on Young's modulus of SCS nanostructures and compare their $E$ results. It was found that different testing methods showed different values of $E$. With a simplified model taking surface effect into account, we qualitatively explained the difference that for Si nanobeams $E_{t}$ measured by tensile test are not the same as $E_{r}$ measured by resonance test, it may realize that the measurement of $E$ in nanoscale is more complex than in macroscale.

\section{Current Experimental Tests on SCS Nanostructures}

Mechanical property measurements of nanostructures, such as nanobeams and nanowires, are very challenging because of the difficulties in (1) sample preparation and nanomanipulating and (2) measuring force and displacement (strain and stress) with nanoscale resolution. But in these years researchers have demonstrated some mechanical tests on SCS nanobeams and nanowires, which cover bending tests, resonance tests, and tensile tests. 
2.1. Bending Tests. Atomic force microscopy (AFM) is usually employed to give 3D image of the topography of the sample surface, control and apply a specified amount of force on the sample. It is suitable to use AFM in mechanical tests on a single- (double-) clamped nanobeam (nanowire) by applying force to the specimen and measure deformation simultaneously. By using beam bending equations, the mechanical properties can be deduced. For double-clamped beam being applied as a force in its middle point, Young's modulus can be expressed as

$$
E=\frac{l^{3}}{192 I} m
$$

where $l$ is the beam/wire length, $I$ is the moment of inertia for the beam cross-section, and $m$ is the gradient of the forcedisplacement curve during bending [5].

Namazu et al. patterned Si nanobeams by means of field-enhanced anodization on SOI wafer using AFM and obtained the nanobeams by anisotropic wet etching, which are $<110>$-oriented with thickness of $255 \mathrm{~nm}$ and widths from 200 to $800 \mathrm{~nm}$. By applying bending tests on these double clamped nanobeams, the measured values of Young's modulus are determined to be $169 \mathrm{GPa}$ on average. These results indicate that the specimen size has no influence on $E$ yet [5]. Sundararajan et al. also followed the experimental procedure and presented Young's modulus values of $182 \pm$ $11 \mathrm{GPa}[6,7]$.

Virwani et al. patterned some $200-400 \mathrm{~nm}$-wide 193/255 nm-thick Si nanobeam on SOI wafer using e-beam lithography, and their bending tests by AFM gave an $\mathrm{Si}$ Young's modulus of $174 \mathrm{GPa}$ along $<100>$ direction [8].

Paulo et al. also used AFM to characterize the mechanical elasticity of their Si nanowires synthesized by VaporLiquid-Solid method. The nanowires are horizontally grown between the two facing $\mathrm{Si}$ sidewalls of microtrenches. The values of $E$ were estimated to be $186 \mathrm{GPa}$ and $207 \mathrm{GPa}$, respectively, for single- and double-clamped Si nanowires [9].

2.2. Resonance Tests. According to Euler-Bernoulli theory, dynamic studies on the resonant frequency of nanobeams and nanowires can provide Young's modulus when the geometry is determined accurately.

In the work of $\mathrm{Li}$ et al. [10], the SCS untratine cantilevers were patterned on SOI wafer and dry etched along $<110\rangle$ crystalline direction with thicknesses ranging from $12-300 \mathrm{~nm}$. Resonant properties of the cantilevers were characterized in high vacuum chamber by laser vibrometer. A pulsed light beam from a laser diode excites the cantilever to resonate and another fiber-coupled laser to pick up the vibration signal quality factor $Q$. The fundamental resonant frequency $f_{0}$ was obtained and Young's modulus was deduced using following equation:

$$
f_{0} \approx 0.162\left(\frac{E}{\rho}\right)^{1 / 2} \frac{\mathrm{t}}{l^{2}}
$$

where $\rho$ is the material density, $t$ is the thickness of the cantilever, and $l$ is the length. The $E$ was fitted to be $68 \mathrm{GPa}$

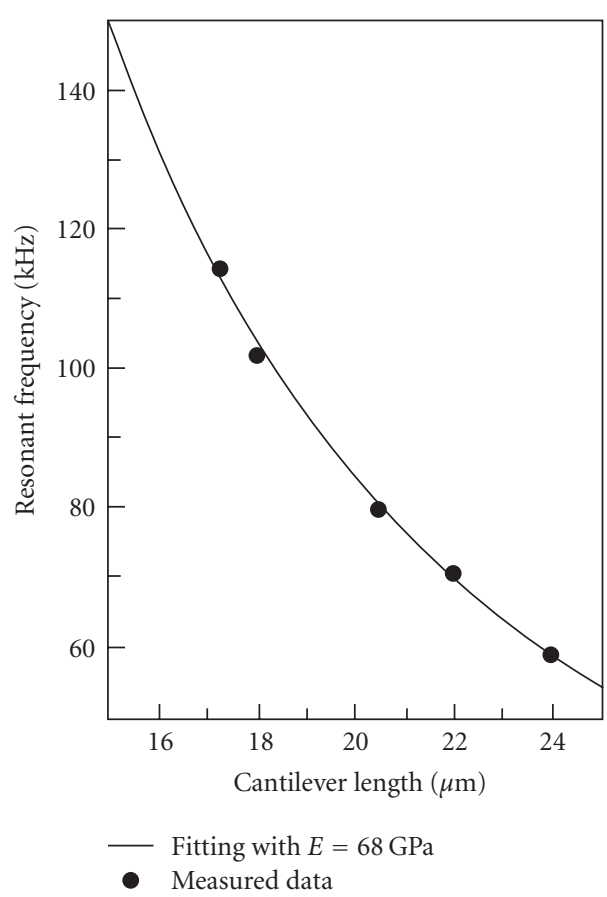

(a)

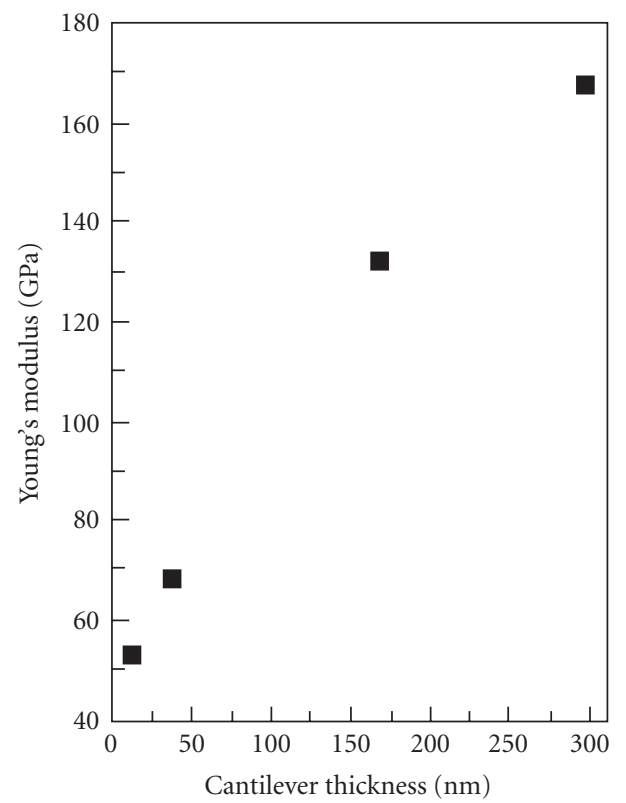

(b)

Figure 1: (a) Resonant frequency data for a set of $38.5 \mathrm{~nm}$ thick cantilevers (with varied length) are fitted into theoretical curve based on resonant frequency expression. Young's modulus of $68 \mathrm{GPa}$ is obtained from the fitted result. (b) The monotonous decrease in Young's modulus value of the ultrathin SCS cantilevers is obtained. The thickness of the cantilevers is in the range of 300-12 nm. (Reprinted with permission from [10]. 2003 American Institute of Physics).

for 38.5-nm-thick cantilever, and $53 \mathrm{GPa}$ for 12-nm-thick cantilever, as shown in Figure 1, it demonstrated that Young's 


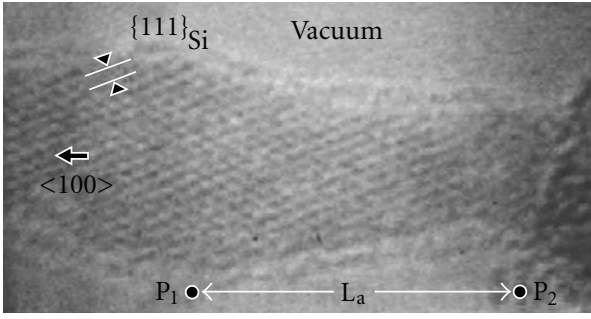

(a)

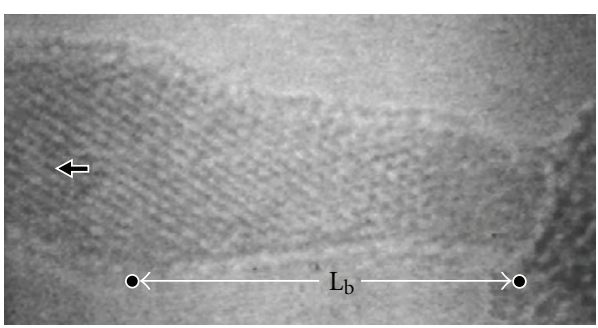

(b)

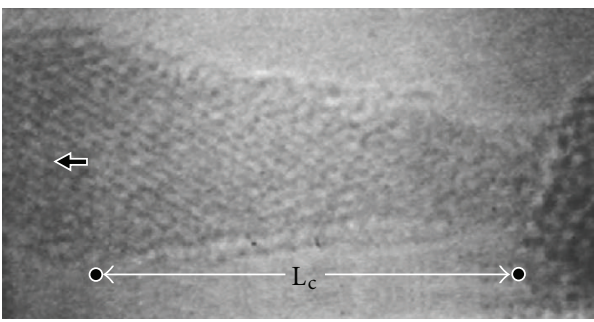

(c)

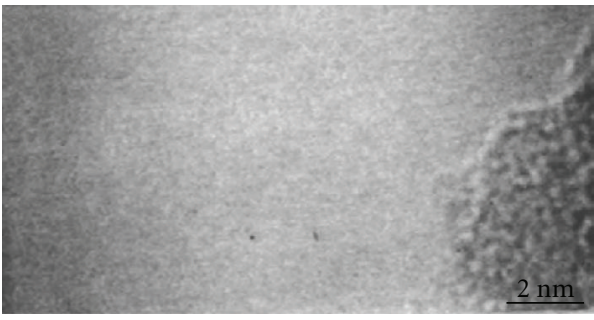

(d)

Figure 2: Time-sequent series of high-resolution images of a tensile deformation process of a single crystalline Si nanowire. The tip of the cantilever (the left-hand side) is retracted toward the left as indicated by the bold arrows. (Reprinted with permission from [11]. 2005 American physical Society).

modulus decreased monotonously as the cantilevers become thinner [10].

2.3. Tensile Tests. Among all the methods of $E$ in nanoscale, tensile test is more challenging since the specimens must be free-standing, clamped at both ends, stretched uniaxially, and measured its elongation with nanometer resolution. To facilitate tensile testing on nanosturctures, various nanomanipulators, based on multiaxes actuation, were designed to work inside scanning electron microscope (SEM) or transmission electron microscopy (TEM). With nanostructure being stretched and tensile force being measured by nanomanipulators, sample elongation being observed by

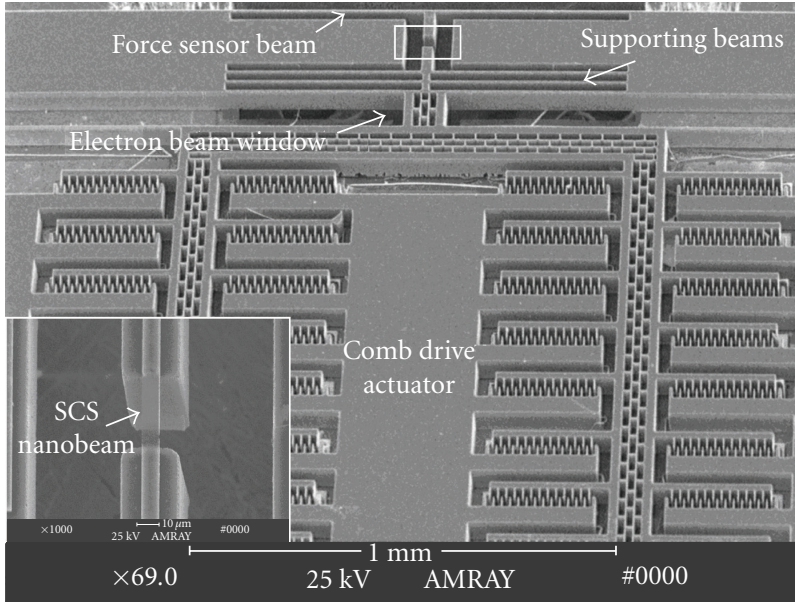

Figure 3: SEM views of the MEMS tensile-testing chip. The structures of beams and combs are shown. Inset is the enlarged view of the sample, seen through the electron beam window. The $<110>$-oriented SCS thin film is connected on its both ends to the supporting structures. (Reprinted with permission from [12]. 2008 IEEE).

SEM or TEM, insitu tensile tests are carried out inside SEM and TEM.

By using a high-resolution TEM equipped inside with nanomanipulators for sub-nano-Newton force measurements (an AFM) and electronic conductance measurements (an STM) [13], Kizuka et al. synthesized $<111>$-oriented Si wires with nanometer widths inside the TEM by nanometertip contact and successive retraction. Then insitu tensile tests were carried out on the nanowire between two tips of AFM and STM. Figure 2 gives time-sequent series of highresolution images of a tensile deformation process of an SCS nanowire with diameter of $4 \pm 2 \mathrm{~nm}$. Young's modulus was found to be $18 \pm 2 \mathrm{GPa}[11]$.

Micro-electro-mechanical systems (MEMSs) can be advantageously employed in the testing of nanoscale samples. It usually consists of three parts: actuator for nanomanipulating sample, sensor for measuring force on the sample, and a cofabricated (or later-attached) sample. By integrating MEMS chip in SEM or TEM, researchers have successfully performed several tensile tests on nanostructures [14-16].

We have designed and fabricated an MEMS tensiletesting chip, a TEM holder with electric terminals, and carried out insitu TEM tensile testing on SCS nanobeam $[12,17]$. The holder and chip fit well in the $0.9 \mathrm{~mm}$ pole piece gap of JOEL 2010. The chip $(5 \mathrm{~mm} \times 9 \mathrm{~mm} \times 0.5 \mathrm{~mm})$ is fabricated by means of Si bulk micromachining and wafer bonding. Figure 3 gives the SEM view of the MEMS chip, with a sample (SCS nanobeam), a comb drive actuator, a force sensor beam, and a TEM electron beam window being integrated into it, and those parts are fabricated in one process. As the electrostatic force actuated comb drive actuator causes no current, it will cause no interference to TEM measurements.

When actuating voltage was applied, with the on-chip comb drive actuator stretching the SCS nanobeam and 


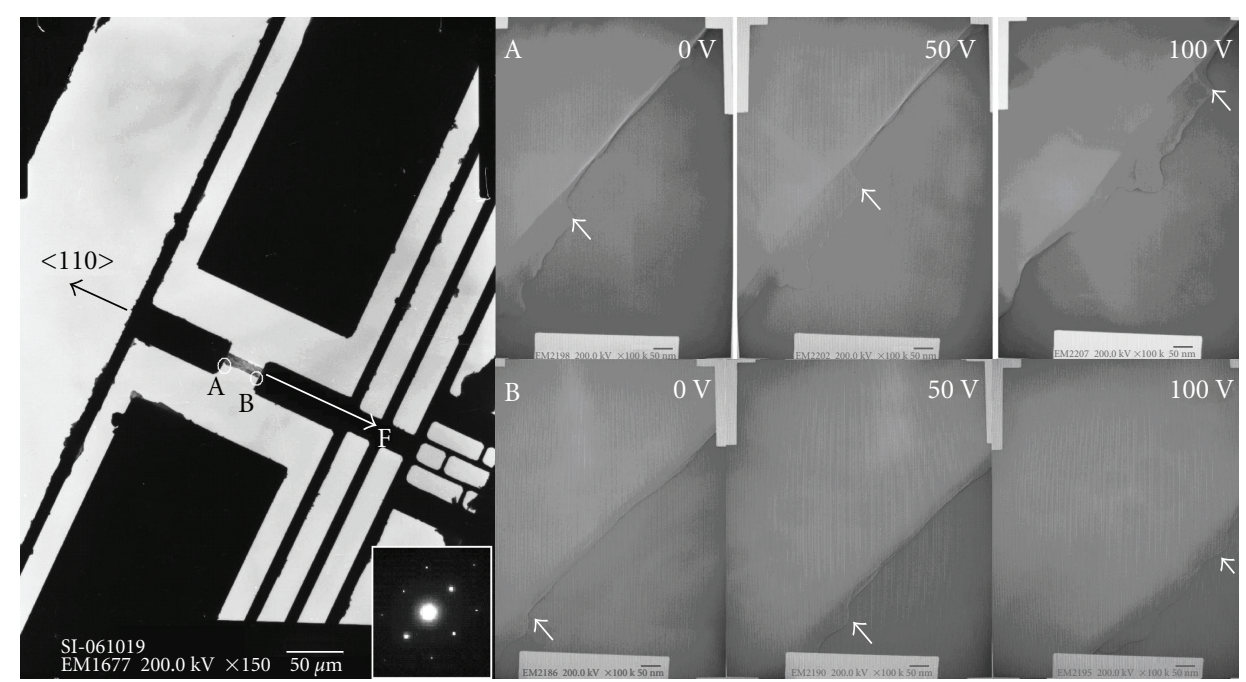

FIGURE 4: TEM bright field (BF) images of the structures and the SAD pattern of the SCS nanobeam (inset) TEM images of the movements of the two ends of the nanobeam, A and B (indicated by arrows). (Reprinted with permission from [12]. 2008 IEEE.)

insitu TEM observation, tensile tests are performed on the $33 \mu \mathrm{m}$-long $8.2 \mu \mathrm{m}$-broad $90 \mathrm{~nm}$-thick <110>-oriented SCS nanobeam. For actuating voltages from $50 \mathrm{~V}$ to $100 \mathrm{~V}$, incremented in $10 \mathrm{~V}$ steps, we snapshot the movements of the two ends of the nanobeam, A and B (indicated by the arrows), as shown in Figure 4. By taking the 4 corner marks, which were the images of 4 fixing film clamps in TEM, as reference positions, we measured the displacements $l_{1}$ (of A) and $l_{2}$ (of B). The elongation of the nanobeam $\Delta l$ was $l_{2}-l_{1}$ and the deflection of the force sensor beam can be considered as $l_{1}$. The tensile force on the SCS nanobeam was calculated as $210 \mathrm{~N} / \mathrm{m} \times l_{1}$, with the elastic constant of the force sensor beam being $210 \mathrm{~N} / \mathrm{m}$. By fitting the strain-stress relationship under different actuating voltages, we obtained a Young's modulus of $167 \mathrm{Gpa}$.

\section{Discussion}

The experimental results on Young's modulus of SCS nanobeams and nanowires are summarized in Table 1 on the catalog of their crystal directions. As the structures dimension decreasing, the resonance test shows a decreasing tendency of Young's modulus on the dimension, which is also shown clearly in Figure 5. The result of $18 \mathrm{GPa}$ obtained by tensile test on an $<111>$-directed SCS nanowire supports this tendency too. All these suggest that for crystal silicon the size effect of $E$ is decreasing as the dimension decreasing.

It is also interesting to note that the measured values of Young's modulus by different testing methods have a little difference. Size effect did not appear at $200 \mathrm{~nm}$-thick nanobeam in bending test, while in resonance test the tendency curve indicates that it should appear at around $250 \mathrm{~nm}$. As for tensile test, Young's modulus still keeps the bulk value at $90 \mathrm{~nm}$-thick nanobeam.

Compared to the tensile test result, Young's modulus by resonance test shows its size effect more early. The different behaviors of $E$ in tensile and resonance test cannot be treated

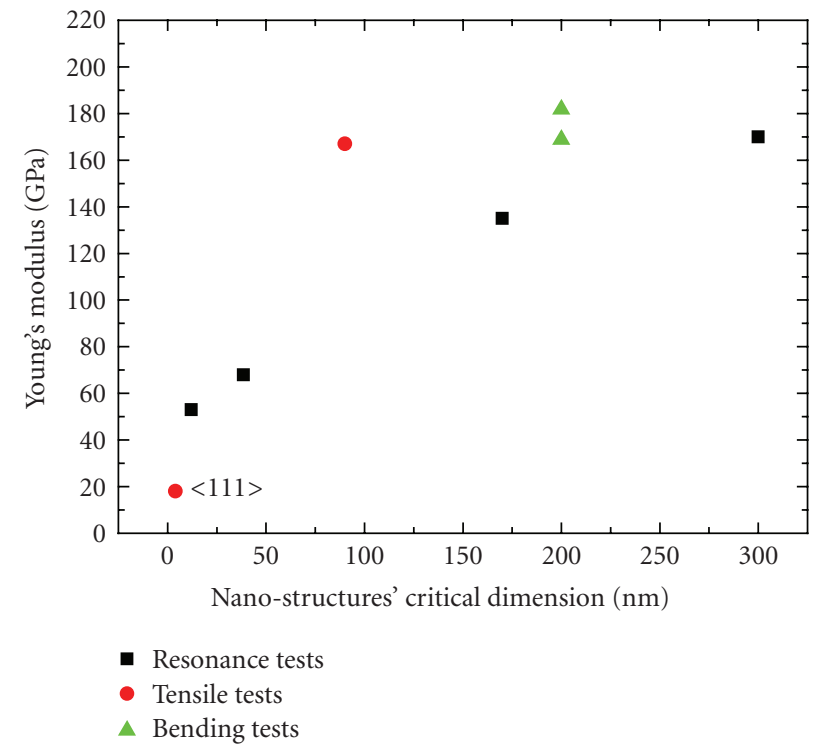

FIGURE 5: Values of Young's modulus versus nanostructures critical dimension, with $E$ measured by bending, resonance, and tensile tests.

as experimental error, and we think it must be surface effect working.

Taking nanobeam, for instance, a model to explain the difference is constructed, in which the nanobeam is treated as a composite beam with a silicon middle layer and two surface layers. The difference of Young's modulus caused by the two different methods can be described as

$$
E_{t}-E_{r}=2\left(1-\frac{E_{s}}{E_{0}}\right)\left(2 \frac{h_{s}}{h}-6 \frac{h_{s}^{2}}{h^{2}}+\frac{h_{s}^{3}}{h^{3}}\right) E_{0},
$$

where $E_{t}$ is Young's modulus of the composite beam by tensile test, and $E_{r}$ is Young's modulus by resonance test [18]. 
TABLE 1: Experimental results of $E$ for SCS nanobeams (nanowires).

\begin{tabular}{|c|c|c|c|c|c|c|}
\hline \multirow{3}{*}{ Direction } & \multicolumn{3}{|c|}{ Dimension $(\mathrm{nm})$} & \multirow{3}{*}{ Young's modulus (GPa) } & \multirow{3}{*}{ Testing method } & \multirow{3}{*}{ Reference } \\
\hline & \multicolumn{2}{|c|}{ Nanobeam } & \multirow{2}{*}{$\begin{array}{l}\text { Nanowire } \\
\text { diameter }\end{array}$} & & & \\
\hline & Width & Thickness & & & & \\
\hline \multirow{7}{*}{$<110>$} & 200 & 255 & - & 169 & Bending & [5] \\
\hline & 200 & 255 & - & 182 & Bending & {$[6,7]$} \\
\hline & N.A. & 300 & - & 170 & Resonance & {$[10]$} \\
\hline & N.A. & 170 & - & 135 & Resonance & {$[10]$} \\
\hline & N.A. & 38.5 & - & 68 & Resonance & {$[10]$} \\
\hline & N.A. & 12 & - & 53 & Resonance & {$[10]$} \\
\hline & 30000 & 90 & - & 167 & Tensile & {$[12,17]$} \\
\hline$<100>$ & 200 & 193 & - & 174 & Bending & [8] \\
\hline$<111>$ & - & - & 4 & 18 & Tensile & {$[11]$} \\
\hline N.A. & - & - & 120 & $186 / 207$ & Bending & [9] \\
\hline
\end{tabular}

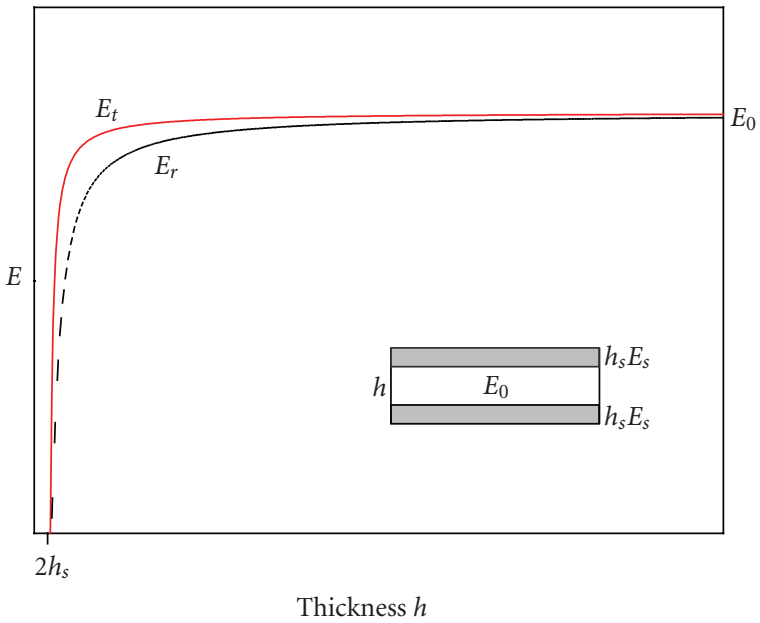

Figure 6: Illustration of the different values of $E_{t}$ (in tensile test) and $E_{r}$ (in resonance test) deduced from a simplified model considering nanobeam as a composite beam with two surfaces of thickness $h_{s}$ and surface $E_{0}$.

Since many theoretical [19-24] and experimental results $[10,11]$ show that the silicon Young's modulus decreases monotonously as the SCS nanostructures dimensions decreasing, it can be deduced that $E_{S}<E_{0}$. The difference between $E_{t}$ and $E_{r}$ is plotted schematically in Figure 6 . When $h$ is much larger than $h_{s}$, every testing method gives a same value $E_{0}$ for Young's modulus. That is the case at macro- or microscale. As $h$ decreases, $E$ decreases, and the value of $E$ by different testing methods will be different from each other. $E_{r}$ shows size effect more early as $h$ decreases, compared with $E_{t}$.

\section{Conclusion}

In this paper, the existing experimental tests of Young's modulus, including bending tests, resonance tests, and tensile tests, on SCS nanostructures are reviewed. The results suggest that at nanoscale, the silicon Young's modulus exhibits obvious size effect of a decreasing tendency on the nanostructures decreasing dimension. Different testing methods may give different Young's modulus values. The size effect of Young's modulus appears earlier in resonance test than in the other methods. By taking surface effect into account, this difference may be qualitatively explained for SCS nanobeam. It indicates that current experiments are still not enough to understand the behavior of SCS Young's modulus at nanoscale thoroughly, more research work should be done for it.

\section{Acknowledgments}

This work is partly financially supported by the National Basic Research Program of China Grant no. 2006CB300403 and the Fund for Creative Research of NSFC Grant no. 60721004 .

\section{References}

[1] K.-K. Lew, L. Pan, T. E. Bogart, et al., "Structural and electrical properties of trimethylboron-doped silicon nanowires," Applied Physics Letters, vol. 85, no. 15, pp. 3101-3103, 2004.

[2] A. Gaidarzhy, G. Zolfagharkhani, R. L. Badzey, and P. Mohanty, "Evidence for quantized displacement in macroscopic nanomechanical oscillators," Physical Review Letters, vol. 94, no. 3, Article ID 030402, 4 pages, 2005.

[3] D. Li, Y. Wu, P. Kim, L. Shi, P. Yang, and A. Majumdar, "Thermal conductivity of individual silicon nanowires," Applied Physics Letters, vol. 83, no. 14, pp. 2934-2936, 2003.

[4] C. T. Huang, C. L. Hsin, K. W. Huang, et al., "Er-doped silicon nanowires with $1.54 \mu \mathrm{m}$ light-emitting and enhanced electrical and field emission properties," Applied Physics Letters, vol. 91, no. 9, Article ID 093133, 3 pages, 2007.

[5] T. Namazu, Y. Isono, and T. Tanaka, "Evaluation of size effect on mechanical properties of single crystal silicon by nanoscale bending test using AFM," Journal of Microelectromechanical Systems, vol. 9, no. 4, pp. 450-459, 2000.

[6] S. Sundararajan, B. Bhushan, T. Namazu, and Y. Isono, "Mechanical property measurements of nanoscale structures using an atomic force microscope," Ultramicroscopy, vol. 91, no. 1-4, pp. 111-118, 2002. 
[7] S. Sundararajan and B. Bhushan, "Development of AFMbased techniques to measure mechanical properties of nanoscale structures," Sensors and Actuators A, vol. 101, no. 3, pp. 338-351, 2002.

[8] K. R. Virwani, A. P. Malshe, W. F. Schmidt, and D. K. Sood, "Fabrication and testing of nanomechanical $<100>$ silicon beam structures using a scanning probe system," in Nano- and Microtechnology: Materials, Processes, Packaging, and Systems, vol. 4936 of Proceedings of SPIE, pp. 50-57, Melbourne, Australia, December 2002.

[9] A. S. Paulo, J. Bokor, R. T. Howe, et al., "Mechanical elasticity of single and double clamped silicon nanobeams fabricated by the vapor-liquid-solid method," Applied Physics Letters, vol. 87, no. 5, Article ID 053111, 3 pages, 2005.

[10] X. Li, T. Ono, Y. L. Wang, and M. Esashi, "Ultrathin singlecrystalline-silicon cantilever resonators: fabrication technology and significant specimen size effect on Young's modulus," Applied Physics Letters, vol. 83, no. 15, pp. 3081-3083, 2003.

[11] T. Kizuka, Y. Takatani, K. Asaka, and R. Yoshizaki, "Measurements of the atomistic mechanics of single crystalline silicon wires of nanometer width," Physical Review B, vol. 72, no. 3, Article ID 035333, 6 pages, 2005.

[12] Q. H. Jin, T. Li, Y. L. Wang, et al., "In-situ TEM tensile test of $90 \mathrm{~nm}$-thick <110> SCS beam using MEMS chip," in Proceedings of the IEEE Conference on Sensors (ICSENS '08), pp. 1116-1118, Lecce, Italy, October 2008.

[13] T. Kizuka, H. Ohmi, T. Sumi, et al., "Simultaneous observation of millisecond dynamics in atomistic structure, force and conductance on the basis of transmission electron microscopy," Japanese Journal of Applied Physics, vol. 40, pp. L170-L173, 2001.

[14] M. A. Haque and M. T. A. Saif, "In-situ tensile testing of nanoscale specimens in SEM and TEM," Experimental Mechanics, vol. 42, no. 1, pp. 123-128, 2002.

[15] M. A. Haque and M. T. A. Saif, "Application of MEMS force sensors for in situ mechanical characterization of nano-scale thin films in SEM and TEM," Sensors and Actuators A, vol. 9798, pp. 239-245, 2002.

[16] Y. Zhu and H. D. Espinosa, "An electromechanical material testing system for in situ electron microscopy and applications," Proceedings of the National Academy of Sciences of the United States of America, vol. 102, no. 41, pp. 14503-14508, 2005.

[17] Q. H. Jin, Y. L. Wang, T. Li, X. Li, and F. Xu, "A MEMS device for in-situ TEM test of SCS nanobeam," Science in China Series E, vol. 51, no. 9, pp. 1491-1496, 2008.

[18] Q. H. Jin, Study on in-situ TEM tensile tests on SCS nanostructures, Ph.D. dissertation, Shanghai Jiao Tong University, Shanghai, China, 2008.

[19] K. K. Mon, "Mechanical properties of model nanostructures," Physical Review B, vol. 50, no. 22, pp. 16718-16721, 1994.

[20] F. Bao, H. Yu, and Q.-A. Huang, "Elastic modulus of nanometer silicon membrane," in Proceedings of IEEE International Conference on Information Acquisition (ICIA '06), pp. 85-90, Weihai, Shandong, August 2006.

[21] B. Lee and R. E. Rudd, "First-principles study of the Young's modulus of Si $<001>$ nanowires," Physical Review B, vol. 75, no. 4, Article ID 041305, 4 pages, 2007.

[22] C. Q. Sun, "Thermo-mechanical behavior of low-dimensional systems: the local bond average approach," Progress in Materials Science, vol. 54, no. 2, pp. 179-307, 2009.

[23] J.-G. Guo and Y.-P. Zhao, "The size-dependent elastic properties of nanofilms with surface effects," Journal of Applied Physics, vol. 98, no. 7, Article ID 074306, 11 pages, 2005.
[24] J.-G. Guo and Y.-P. Zhao, "The size-dependent bending elastic properties of nanobeams with surface effects," Nanotechnology, vol. 18, no. 29, Article ID 295701, 6 pages, 2007. 

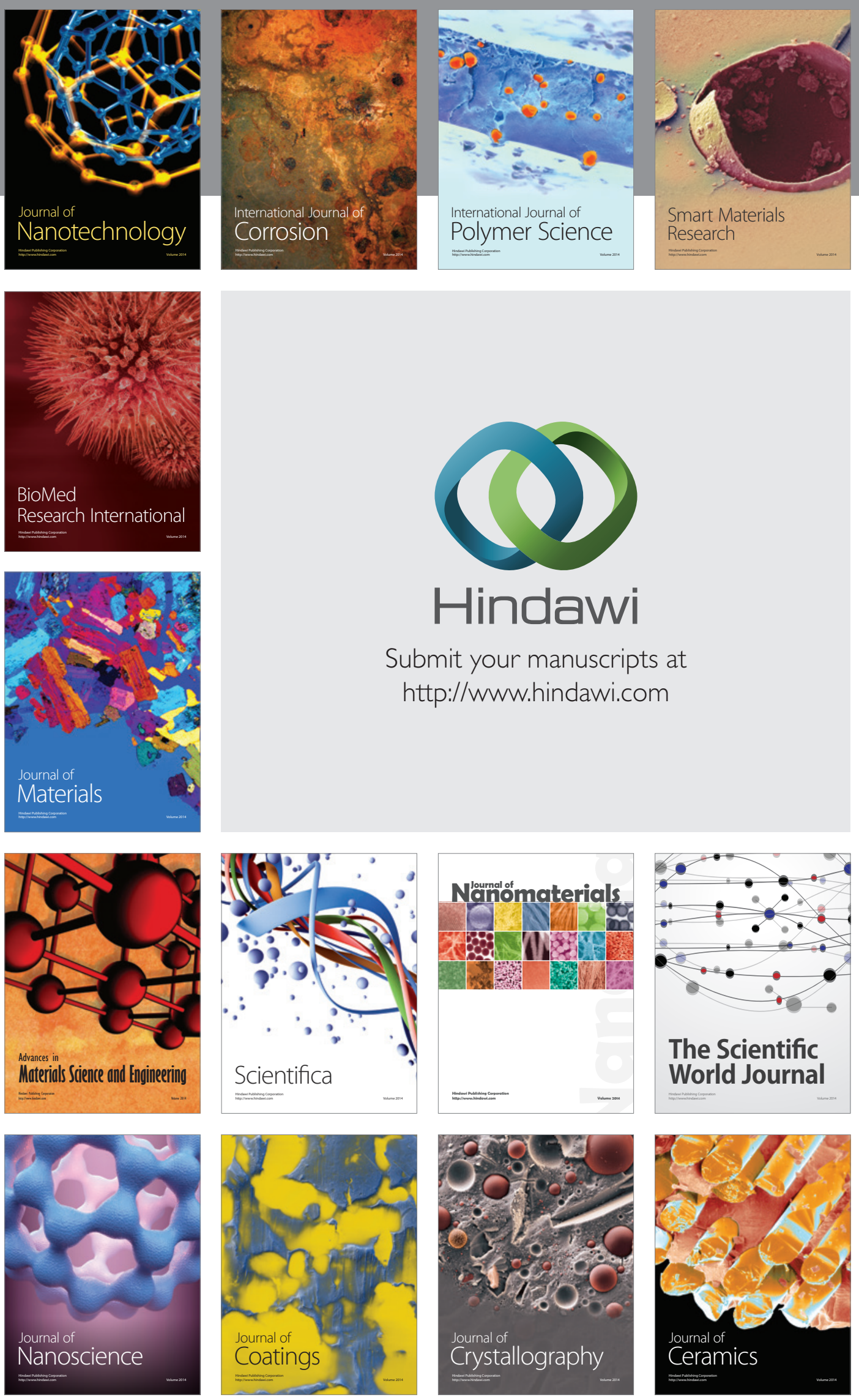

The Scientific World Journal

Submit your manuscripts at

http://www.hindawi.com

\section{World Journal}

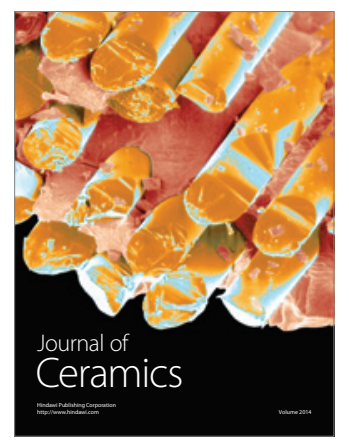

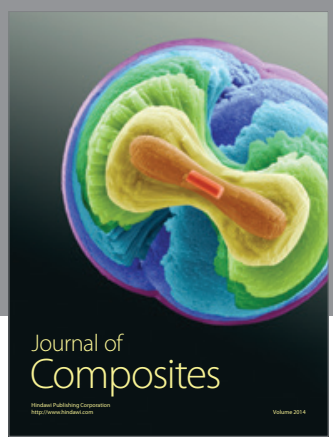
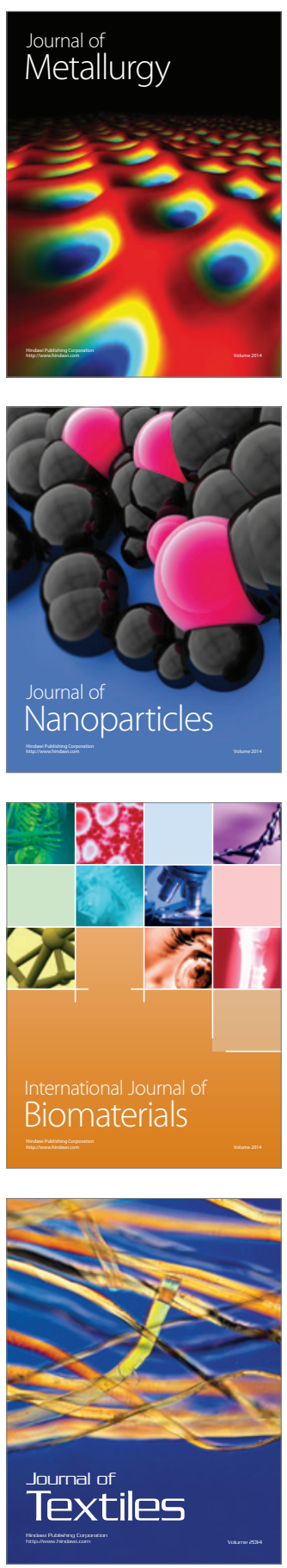